myocardial segments for detecting artefacts, but with indicating distractions leading to suboptimal accuracy (figure 1c). A $\mathrm{CNN}$ trained with additional guidance on attention is shown to pay the desired attention to the right structures and avoids distractions (figure 1d).

Conclusion CNN designed with support of attention visualisation, and trained with guidance on attention can lead to significantly more transparent and accountable AI use in clinical practice.

\section{INTER-FIELD STRENGTH AGREEMENT OF MYOCARDIAL STRAIN MEASUREMENTS USING TISSUE TRACKING CARDIOVASCULAR MAGNETIC RESONANCE IMAGING}

S Ayton, L Athithan, KS Parke, JV Wormleighton, C Budgeon, GP McCann, GS Gulsin. Department of Cardiovascular Sciences, University of Leicester, and NIHR Leicester Biomedical Research Centre, Glenfield Hospital, Leicester, UK

\subsection{6/heartjnl-2019-BSCMR.10}

Funding acknowledgement The participants included in this study were recruited as part of a larger study funded by the National Institute for Health Research Career Development Fellowship (CDF 2014-07-045).

Background The agreement of left ventricular (LV) strain measurements using cardiovascular magnetic resonance (CMR) tissue tracking at different field strengths is unknown. We compared inter-field strength agreement of systolic and diastolic strain/strain rates at $1.5 \mathrm{~T}$ and $3 \mathrm{~T}$.

Methods We prospectively enrolled 17 healthy volunteers and 3 participants with diabetes but no cardiovascular disease, from a diet and exercise intervention study. Subjects underwent CMR balanced steady state free precession cine imaging with retrospective ECG gating at $3 \mathrm{~T}$ followed by $1.5 \mathrm{~T}$ on the same day. Acquisition voxel size was $1.66 \times 1.33 \times$ $8 \mathrm{~mm}$ and $1.90 \times 1.52 \times 8 \mathrm{~mm}$, respectively, temporal resolution $48 \mathrm{~ms}$ and $38 \mathrm{~ms}$, TR 3.44 and 2.76 and TE 1.51 and 1.15. Acquisitions were reconstructed to 30 phases. Short axis cine images at $1.5 \mathrm{~T}$ were obtained following adenosine stress and gadolinium contrast, whereas short axis images at $3 \mathrm{~T}$ and all long axis images were acquired before contrast administration. Scans were analysed offline by a single experienced observer blinded to patient details, using cmr42 software (Circle Cardiovascular Imaging, Calgary, Canada). End-diastolic epicardial and endocardial and end-systolic endocardial borders were defined manually and propagated by the software throughout the cardiac cycle to derive longitudinal, circumferential and radial global systolic strain (GLS, GCS and GRS) and peak early diastolic strain rates (longitudinal, circumferential and radial PEDSR). Inter-field strength
Abstract 10 Table 1 Left ventricular function, volume and strain measurements

\begin{tabular}{llll}
\hline \multicolumn{2}{l}{ LV function and volume } & & \\
\hline LVEF (\%) & $59.8(4.1)$ & & \\
LVEDV (ml) & $158.6(28.1)$ & & \\
LVESV (ml) & $64(14.1)$ & & ICC \\
LV mass (g) & $87.1(21)$ & $3 T$ & 0 \\
LV strain measurements & $1.5 T$ & $-15.6(1.4)$ & 0.49 \\
GLS (\%) & $-17.5(1.4)$ & $-18.4(2.3)$ & 0.49 \\
GCS (\%) & $-19.8(2)$ & $31.7(6.7)$ & 0 \\
GRS (\%) & $35.3(6.1)$ & $0.72(0.1)$ & 0.3 \\
Longitudinal PEDSR $\left(\mathrm{s}^{-1}\right)$ & $0.87(0.14)$ & $0.93(0.21)$ & 0.12 \\
Circumferential PEDSR $\left(\mathrm{s}^{-1}\right)$ & $1.03(0.14)$ & $-1.76(0.56)$ & \\
Radial PEDSR $\left(\mathrm{s}^{-1}\right)$ & $-2.06(0.42)$ & & \\
\hline
\end{tabular}

mean (SD): LV, left ventricle; LVEF, left ventricular ejection fraction, LVEDV, left ventricula end diastolic volume; LVESV, left ventricular end systolic volume; GLS, global longitudinal strain; GCS, global circumferential strain GRS, global radial strain; PEDSR, peak end diastolic strain rate; ICC, intraclass correlation coefficient

agreement was assessed with intraclass correlation (ICC) and Bland Altman plots.

Results Mean age was $50 \pm 6.3$ years and 50\% were male. LV function, volumetric and strain data are presented in table 1. Mean systolic strain measurements were higher at $1.5 \mathrm{~T}$ versus 3T (all $\mathrm{p}<0.05$ ). Inter-field strength agreement was fair for GCS and GRS (ICC=0.49). Despite all images being acquired before contrast, there was no agreement for GLS (ICC $=0$; table 1). Bland Altman plots are shown in figure 1. All PEDSR measurements had poor agreement (ICC range $0-0.3$; table 1).

Conclusion In this pilot study, strain measurements showed fair inter-field strength agreement for GCS and GRS and no agreement for GLS. This may relate to different signal-to-noise characteristics between field strengths. Further work is needed with randomised comparisons, but these data suggest strain measurements obtained at $1.5 \mathrm{~T}$ and $3 \mathrm{~T}$ are not interchangeable.

\section{NOVICE MARATHON TRAINING REVERSES VASCULAR AGEING}

${ }^{1,2} \mathrm{AN}$ Bhuva, ${ }^{3} \mathrm{~A}$ D'Silva, ${ }^{2,4} \mathrm{C}$ Torlasco, ${ }^{1} \mathrm{~S}$ Jones, ${ }^{1} \mathrm{~N}$ Nadarajan, ${ }^{2} \mathrm{~J}$ Van Zalen, ${ }^{2} \mathrm{R}$ Boubertakh, ${ }^{1} \mathrm{~N}$ Chaturvedi, ${ }^{2} \mathrm{G}$ Lloyd, ${ }^{3} \mathrm{~S}$ Sharma, ${ }^{1,2} \mathrm{~J} \mathrm{C}$ Moon, ${ }^{1} \mathrm{AD}$ Hughes, ${ }^{1,2} \mathrm{CH}$ Manisty. 'Institute for Cardiovascular Science, University College London, London, UKi ${ }^{2}$ Department of Cardiovascular Imaging, Barts Heart Centre, Barts Health NHS Trust, London, UK; ${ }^{3}$ Cardiology Clinical and Academic Group, St George's, University of London, London, UK; ${ }^{4}$ Istituto Auxologico Italiano, IRCCS, Dept of Cardiovascular, Neural and Metabolic Sciences, San Luca Hospital, Italy

\subsection{6/heartjnl-2019-BSCMR.11}
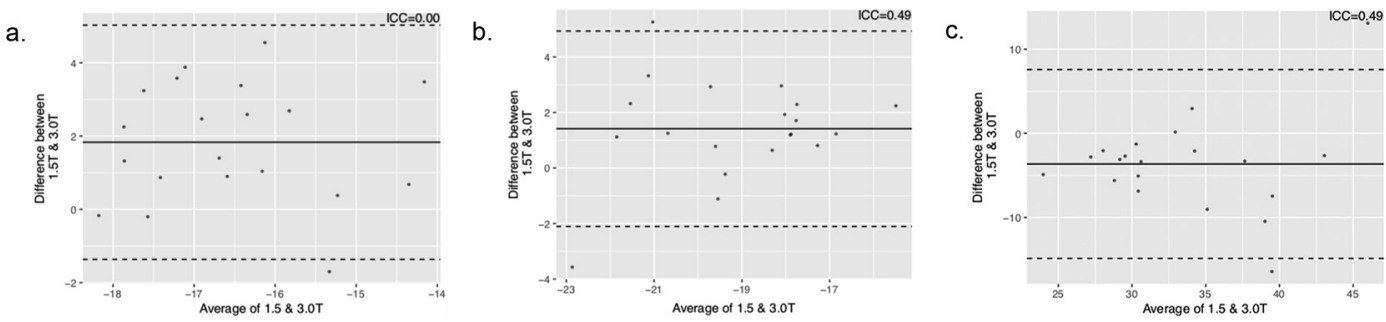

Abstract 10 Figure 1 Bland Altman plots for systolic strain measurements; a. global longitudinal strain (GLS), b. global circumferential strain (GCS), c. global radial strain (GRS) 